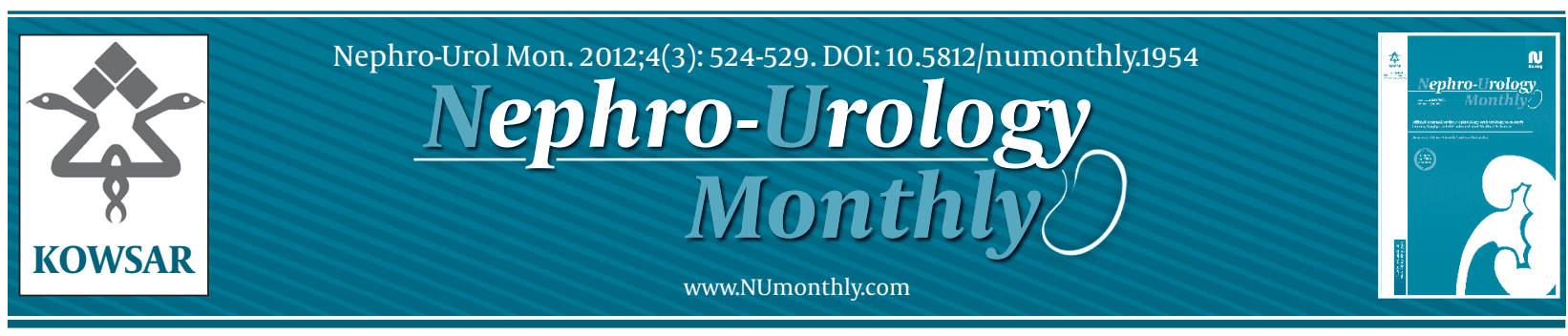

\title{
Lessons From the KK-Ay Mouse, a Spontaneous Animal Model for the Treatment of Human Type 2 Diabetic Nephropathy
}

\author{
Yasuhiko Tomino ${ }^{1^{*}}$ \\ ${ }^{1}$ Division of Nephrology, Department of Internal Medicine, Juntendo University Faculty of Medicine, Tokyo, Japan
}

\begin{tabular}{l}
\hline A R T I C L E I N F O \\
\hline Article type: \\
Review Article \\
\hline Article history: \\
Received: 27 Jul 2011 \\
Revised: 06 Sep 2011 \\
Accepted:15 Sep 2011 \\
\hline
\end{tabular}

Keywords:

Models, Animal

Therapeutics

Diabetic Nephropathies

\begin{abstract}
A B S T R A C T
Diabetic nephropathy is a major cause of end-stage kidney disease (ESKD) in patients with type 1 and type 2 diabetes throughout the world. In human glomeruli, expansion of diffuse mesangial matrices, exudative lesions and/or segmental nodular sclerosis are pathological features of diabetic nephropathy. There have been many reports on the pathogenesis and treatment of type 2 diabetes using various animal models.

It appears that KK-Ay mice, especially in terms of their immunohistological findings, are a suitable animal model for human type 2 diabetic nephropathy. Many compounds have been reported to be advanced glycation end product (AGE) inhibitors such as aminoguanidine, angiotensin II receptor inhibitors and pyridoxamine, and these are useful in therapeutic interventions for reducing AGEs. Pyridoxamine ameliorates lipid peroxidation and insulin resistance in KK-Ay mice. Combination therapy with angiotensin converting inhibitors (ACE-I) and angiotensin II type 1 receptor blockers (ARB), including an $A R B$ and 1,25-dihydroxyvitamin D3, i.e. anti-hypertensive and anti-reactive oxygen species effects, or with eicosapentaenoic acid (EPA), i.e. anti-microinflammation effect, have shown efficacy in the treatment of diabetic nephropathy in KK-Ay mice. It appears that KK-Ay mice are a useful spontaneous animal model for the evaluation of pathogenesis and treatment in patients with type 2 diabetic nephropathy.
\end{abstract}

Copyright @ 2012 Kowsar Corp. All rights reserved.

- Implication for health policy/practice/research/medical education:

KK-Ay mouse is a useful spontaneous animal model for type 2 diabetic nephropathy.

Please cite this paper as:

Tomino Y. Lessons From the KK-Ay Mouse, a Spontaneous Animal Model for the Treatment of Human Type 2 Diabetic Nephropathy. Nephro-Urol Mon.4(3): 524-9. DOI:10.5812/numonthly.1954

\section{Background}

Diabetic nephropathy is a major cause of end-stage kidney disease (ESKD) in patients with type 1 and type 2 diabetes throughout the world. Almost $30 \%$ of diabetic patients in Japan develop nephropathy despite strict blood glucose and blood pressure control. It has been postulated that the initiation of this disease might be

\footnotetext{
* Corresponding author: Yasuhiko Tomino, Division of Nephrology, Department of Internal Medicine, Faculty of Medicine, Juntendo University, 113-8421, Tokyo, Japan. Tel: +81-358021064, Fax: +81-338131183. E-mail: yasu@ juntendo.ac.jp
}

DOI:10.5812/numonthly.1954

Copyright $\odot 2012$ Kowsar Corp. All rights reserved. due to genetic factors. In human glomeruli, expansion of diffuse mesangial matrices, exudative lesions and/or segmental nodular sclerosis are pathological features of diabetic nephropathy. There are many progressive factors in patients with diabetic nephropathy, but no specific treatment for human diabetic nephropathy based on the initiative and progressive factors that have been found. Thus, it is important to determine both the pathogenesis and potential treatment options using various animal models of type 2 diabetes (Box).

\section{Objectives}

The objectives of this review are 1) to show the charac- 
teristics of the KK-Ay mouse and 2) to introduce strategies for the treatment of type 2 diabetic nephropathy using this spontaneous animal model.

\section{Characteristics of KK-Ay Mouse}

\subsection{Immunohistopathological Findings}

The KK/Ta mouse, a model of type 2 diabetic nephropathy, originated from Japanese native mice as an inbred mouse by Kondo et al. in 1957 (1). This mouse strain is generally considered to be a polygenic disease model. Male KK/Ta mice spontaneously exhibit type 2 diabetes associated with hyperglycemia, glucose intolerance, hyperinsulinemia, mild obesity and microalbuminuria, conditions which are more severe than those found in the female (2-4). Since phenotypic characteristics of the KK/Ta mouse are not especially marked, the KK-Ay mouse was established by Nishimura et al. (5). This mouse was produced by transferring the yellow obese gene (Ay allele) into the KK/Ta mouse. KK-Ay mice exhibit obesity and hyperglycemia, including high levels of HbA1c and albuminuria (5). The KK-Ay mouse strain was established in 1969, and these mice are widely used as an experimental model for type 2 diabetes mellitus.

Pathological changes in the glomeruli of KK-Ay mice were consistent with those found in the early stages of human diabetic nephropathy (6). The renal histological changes, i.e. diffuse mesangial expansion with mesangial cell proliferation and segmental sclerosis in KK-Ay mice, are more severe than those which develop in $\mathrm{KK} /$ Ta mice (Figures 1 and 2) (Table) (6). Linear staining of IgG and diffuse thickening were observed in the glomerular capillary walls. Advanced glycation end products (AGE) and transforming growth factor-beta (TGF- $\beta$ ) protein appeared to be localized in the glomerular mesangial areas (7). The mice also develop renal lesions that closely resemble those seen in human diabetic nephropathy. It appears that KK-Ay mice, especially in terms of their histopathological findings, are a suitable animal model for the early stages of type 2 diabetic nephropathy.

\subsection{Podocyte Loss and Glomerulosclerosis}

Morphometric analysis has contributed greatly to our understanding of diabetic nephropathy. Generally, the progression of nephropathy is associated with a reduction in the number of podocytes per glomerulus. Remaining podocytes are obliged to grow and to extend their foot processes in order to maintain the area that they cover. The authors previously reported that podocyte injury might provide additional prognostic information in patients with IgA nephropathy, the most common chronic glomerulonephritis, examining a small amount of renal biopsy tissue (8). Lemley et al. (9) reported podocytopenia (podocyte loss) and disease activity in patients with IgA nephropathy. However, the glomerular structure in type 2 diabetes has been studied less extensively even though this form of diabetes is a more common cause of ESKD. Macedo et al. (10) reported that control of hyperglycemia prevented the glomerular basement membrane (GBM) from thickening as well as reducing the number of podocytes in the early and late (12 months) stages of alloxan induced diabetic nephropathy. To determine the number of podocytes, the authors analyzed glomerular lesions in KK-Ay mice at 20 weeks of age through morphometric analysis. Levels of urinary protein were also measured. Glomerular enlargement and mesangial expansion were observed in KK-Ay mice at 20 weeks of age. The mean number of podocytes per glomerulus (NG pod) in diabetic KK-Ay mice was significantly lower than that seen in non-diabetic BALB/cA mice. Mean NG pod/ glomerular volume (GV) per glomerulus was also significantly decreased in the diabetic KK-Ay mice. Apoptotic bodies in the podocytes were observed by the tunnel method. The level of urinary albumin/creatinine (ACR) in the diabetic KK-Ay mice was significantly higher than that found in non-diabetic BALB/cA mice at 20 weeks of age. It appears that podocyte loss might induce albuminuria in KK-Ay mice (Ishikawa, et al. JDM, inpress).

\subsection{Analysis of Candidate Genes}

Pathogenesis and the development of human diabetic nephropathy involve genetic factors. Since human diabetic nephropathy is a heterogeneous disorder, the identification of the responsible gene loci is difficult. Aoki et al. (11) studied candidate gene loci for diabetic nephropathy using quantitative trait locus (QTL) analysis of the spontaneous animal model for diabetic nephropathy KK-Ay $\times$ normal BALB/CA F2 intercross mice. Urinary ACR, HbA1c and fasting body weights (FBW) at 8, 12, 16 and 20 weeks were examined in $270(\mathrm{KK}-\mathrm{Ay} \times \mathrm{BALB} / \mathrm{CA}) \mathrm{F} 2$ intercross mice. Genotypes were investigated by QTL analysis using 86 microsatellite markers. ACR in mice at 20 weeks and ACR gain showed a suggestive linkage to chromosome 9 (LOD: 3.8 and 3.4 respectively; designed ACR-1). Gene loci contributing to HbA1c indicated a significant linkage to chromosome 7 (LOD: $5.8,8.9$ ) in mice at 8 and 20 weeks (designed HbA1c-1), and FBW indicated a significant linkage to chromosome 1 (LOD: $5.5,5.2$ ) in mice at 8 and 12 weeks (designed Fbw-1). At 20 weeks, the glomerular/Bowman's capsule volume (G/B) ratio of F2 mice homozygous BB for D9Mit66 was significantly higher than that seen in the homozygous KK and heterozygous KB F2 progeny. Pancreatic islets in homozygous KK and heterozygous KB found in the D7Mit100 F2 progeny were larger than those in the homozygous BB F2 progeny. QTL analysis of the KK-Ay mice revealed several new loci contributing to diabetic nephropathy and related phenotypes. Thus, it appears that type 2 diabetes and diabetic nephropathy in KK-Ay mice have different genetic factors (11). 
Figure 1. Light Microscopical Findings of a Glomerulus from a KK-Ay/Ta Mouse (PAS Staining, x400)

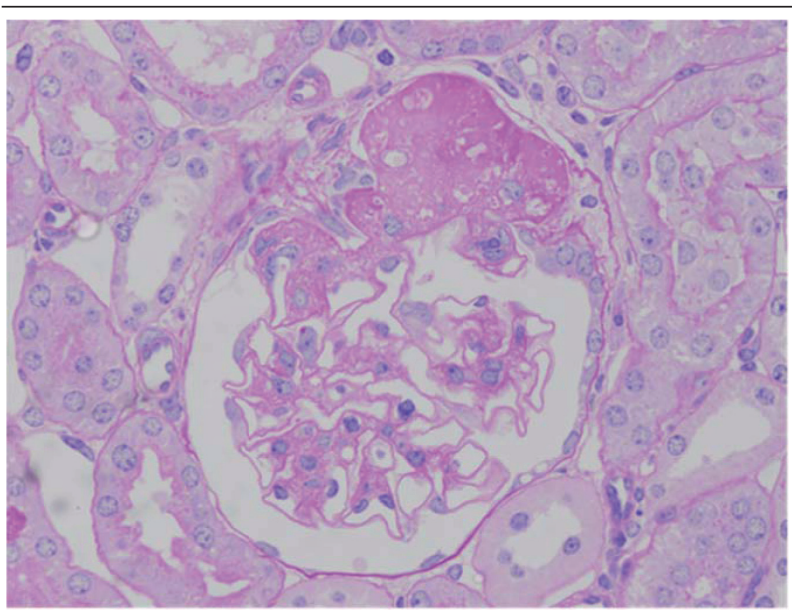

The renal histological changes, i.e. diffuse mesangial expansion with mesangial cell proliferation and segmental sclerosis, in the KK-Ay/Ta mouse are more severe than those seen in the KK/Ta mouse.

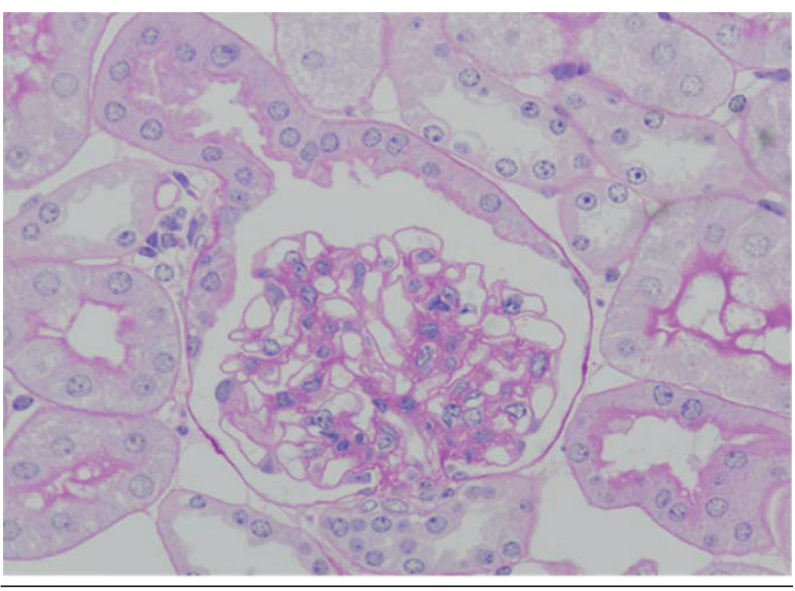

Figure 2. Light Microscopical Findings of a Glomerulus from a $\mathrm{KK} / \mathrm{Ta}$ Mouse (PAS Staining, x400)

\section{Approaches to the Treatment of Type 2 Diabetic Nephropathy}

4.1.Anti-Glycation and Anti-Reactive Oxygen Species Effects of Pyridoxamine (K-163)

Non-enzymatic glycation has been implicated in the pathogenesis of diabetic nephropathy. There are multiple pathways for the formation of AGEs, including $\mathrm{N} \varepsilon$ - carboxymethyllysine (CML), Ne-carboxyethyllysine (CEL) and pentosidine, from glucose, antioxidation products of glucose, Schiff bases and Amadori products. The presence of AGEs is closely related to hyperglycemia and their pathobiochemistry could explain diabetic nephropathy (12). Specific AGEs, such as CML, are major products of glycoxidation reactions. In therapeutic interventions to reduce AGEs, many compounds have been reported to be effective AGE inhibitors, such as aminoguanidine, phenacyl thiazolium bromide, 2-isopropylidenehydrazono-4-oxo-thiazolidine-5-yl-acetanilide (OPB-9195), 2,3-diaminophenazine, vitamin $\mathrm{C}$, vitamin $\mathrm{E}$, angiotensin II receptor inhibitors and pyridoxamine (12).

Pyridoxamine was introduced by Khalifah et al. (13) as an inhibitor of AGE formation from Amadori products. Degenhardt et al. (14) reported that pyridoxamine inhibited AGE formation and retarded the development of diabetic nephropathy in streptozotocin treated rats, an animal model for type 1 diabetes mellitus. In 2007, my colleagues Tanimoto et al. (15), reported that the development of type 2 diabetic nephropathy could be prevented in KK-Ay mice by the use of pyridoxamine (K-163), an AGE inhibitor. AGEs have been associated with increased oxidative and nitrosative stresses in both in vitro and in vivo studies. Pyridoxamine, especially at $400 \mathrm{mg} / \mathrm{kg}$ body weight per day, improved levels of urinary ACR, fasting serum triglyceride (TG) and 3-deoxyglucosone (3DG) in KK-Ay mice. CML and nitrotyrosine accumulation in the glomeruli were decreased. TGF- $\beta 1$ and laminin- $\beta 1$ messenger RNA expressions in the kidneys were significantly lower than those in the controls. The effect of pyridox-

\begin{tabular}{l}
\hline Box. Spontaneous Mouse Model for Type 2 Diabetic Nephropathy \\
\hline KK/Ta mouse \\
KK-Ay mouse \\
Diabetes (db/db) mouse \\
Obese (ob/ob) mouse \\
Fat (fat) mouse \\
Nagoya shibata yasuda (NSY) mouse \\
Tsumura suzuki obese diabetes (TSOD) mouse \\
Akita (Ins2) mouse \\
Tubby (tub) mouse \\
Agouti yellow (Ay) mouse \\
New Zealand obese (NZO) mouse
\end{tabular}

\begin{tabular}{llllll}
\hline Table. Glomerular Changes by Light and Electron Microscopy in KK/Ta and KK-Ay Mice \\
\hline \multicolumn{7}{l}{ Age, $\mathbf{W k}$} & Diffuse Mesangial Expansion & Segmental Sclerosis & GBM $^{\mathbf{a}}$ Thickening & Age, Wk \\
\hline KK/Ta mouse & $16-20$ & $+^{\mathrm{b}}$ & - & + & KK/Ta mouse \\
KK-Ay mouse & 20 & ++ & - & ++ & KK-Ay mouse \\
\hline
\end{tabular}

\footnotetext{
a Abbreviation: GBM, glomerular basement membrane

b $(-)$, negative; $(+)$, mild; $(++)$, moderate
} 
amine was related to improvement of CML and nitrotyrosine accumulation in the kidneys by the anti-AGE and/ or antioxidant effects (15).

Furthermore, Murakoshi et al. (16) reported the pleiotropic effect of pyridoxamine on diabetic complications via CD 36 expression in KK-Ay mice. CD36 is an $88-\mathrm{kDa}$ membrane glycoprotein belonging to the class B scavenger receptor family, which possesses one long extracellular loop between the two transmembrane domains. Pyridoxamine decreased levels of serum TG, especially VLDL, and fasting serum insulin. Accumulation of malondialdehyde (17), an advanced lipoxidation end product, in the pyridoxamine treated group was significantly lower than that in the untreated group. CD 36 accumulation and mRNA expression in the kidneys and adipose tissues in the treatment group were significantly higher than those in the untreated group. It appears that pyridoxamine ameliorated lipid peroxidation and insulin resistance in KK-Ay mice. This pleiotropic effect of pyridoxamine was related to CD36 expression in the kidneys and adipose tissues (16).

Clinically, the phase 2 clinical results demonstrated that the AGE blocker pyridoxamine is generally safe and well tolerated in patients with kidney disease caused by diabetes mellitus (18).

\subsection{Anti-Hypertensive and Anti-Reactive Oxygen Spe- cies Effects}

\subsubsection{Combination Therapy With an ACE (Angiotensin Converting Enzyme) Inhibitor (ACE-I) and an ARB (An- giotensin II Type 1 Receptor Blocker)}

Systemic blood pressure is an important factor associated with the progression of diabetic nephropathy. It is generally considered that ACE-I and/or ARB have a renoprotective effect independent of systemic hypertension. Adenosine monophosphate activated protein kinase (AMPK) has a protective effect on lipid peroxidation. Adiponectin and AMPK might have a role in the pathogenesis of diabetic nephropathy. Blocking the renin-angiotensin system (RAS) increases adiponectin levels and reduces oxidative stress. Yamazaki et al. (19) examined lipid peroxidation via adiponectin and AMPK activation in the kidneys of KK-Ay mice with RAS inhibitors, such as enalapril and/or losartan. KK-Ay mice were given enalapril $(2.5 \mathrm{mg} / \mathrm{kg} /$ day) and/or losartan $(25 \mathrm{mg} / \mathrm{kg} /$ day), or hydralazine $(25 \mathrm{mg} / \mathrm{kg} /$ day $)$ in their drinking water for 8 weeks starting at 8 weeks of age. They were divided into five groups as follows: enalapril $2.5 \mathrm{mg} / \mathrm{kg} /$ day treatment group ( $\mathrm{n}=5$ ), losartan $25 \mathrm{mg} / \mathrm{kg} /$ day treatment group $(\mathrm{n}=5)$, enalapril $2.5 \mathrm{mg} / \mathrm{kg} /$ day + losartan $25 \mathrm{mg} / \mathrm{kg} /$ day combination treatment group $(\mathrm{n}=5)$, hydralazine $25 \mathrm{mg}$ / $\mathrm{kg} /$ day treatment group $(\mathrm{n}=5)$ and tap water group as in the untreated group $(n=5)$. The urinary ACR, serum adiponectin and systemic blood pressure were measured as test parameters. Expressions of adiponectin, phosphoAMPK (p-AMPK) and phospho-acetyl CoA carboxylase (p-ACC) in the kidneys were evaluated through Western blot analyses. Pathological changes of glomeruli were also evaluated by light microscopy. Accumulations of NE-carboxymethyllysine (CML), malondialdehyde (17) and 4-hydroxy-2-nonenal (4-HNE) in the glomeruli were evaluated by immunohistochemical analyses. Enalapril and/or losartan improved levels of urinary ACR with activation of adiponectin, p-AMPK and p-ACC in the kidneys. CML, MDA and 4-HNE expressions in the glomeruli were suppressed significantly with enalapril and/or losartan, especially in the combination treatment groups. In the hydralazine treatment group, the levels of urinary ACR and accumulation of CML/MDA did not improve although the systolic blood pressure decreased. Therefore, it was suggested that the renoprotective effects of ACE and/or ARB are related not only to systemic blood pressure but also to multiple other factors, some of which might be associated with adiponectin, AMPK and ACC activity. It appears that enalapril and/or losartan, especially in combination, inhibits the accumulation of $\mathrm{CML} /$ MDA/4-HNE in diabetic renal tissues. These effects might be related to lipid peroxidation via tissue-specific activation of adiponectin and AMPK (19).

Clinically, Jenning et al. (20) reported the results of a meta-analysis, which suggested that ACEI + ARB reduced 24-hr proteinuria to a greater extent in combination than with ACEI alone.

\subsection{Combination Therapy With ARB and 1,25-dihy- droxyvitamin D3}

Several studies have suggested that the RAS is one of the major mediators for the progression of diabetic nephropathy. ARB is widely used in patients with diabetic nephropathy. However, ARB causes a compensatory renin increase due to the disruption of feedback inhibition in renin production. Recent studies have demonstrated that renin upregulates TGF-b1 and matrix proteins through the renin/prorenin receptors, independent of angiotensin II, which is regulated by extracellular-signal regulated kinase 1 and 2 (ERK1/2), a mitogen-activated protein kinase (MAPK) $(21,22)$. Kaneshiro et al. (23) reported that the human renin/prorenin receptor elicits slow progressive nephropathy through angiotensin II independent MAPKs activation, and initiation of glomerulosclerosis with increased TGF-b1 expression in human renin/prorenin receptor overexpressed transgenic rats.

On the other hand, calcitriol, 1,25-dihydroxyvitamin D3 $(1,25(\mathrm{OH}) 2 \mathrm{D} 3)$ and its analogs have been shown to have therapeutic potential in attenuating experimentally induced kidney diseases (24-29). Schwarz et al. (25) reported that calcitriol treatment suppresses the progression of glomerulosclerosis and albuminuria in subtotally nephrectomized rats. Makibayashi et al. (26) reported that 22-oxacalcitriol, one of the 1,25( $\mathrm{OH}) 2 \mathrm{D} 3$ analogs, has 
been shown to reduce both mesangial cell proliferation and glomerulosclerosis in anti-Thy-1 glomerulonephritis in rats. 1,25(OH)2D3 is a negative endocrine regulator of RAS and suppresses renin biosynthesis $(27,28)$. These studies provide a molecular basis to explore the potential of $1,25(\mathrm{OH}) 2 \mathrm{D} 3$ as a renin inhibitor to control RAS (27). Therefore, it is postulated that combination therapy with ARB and 1,25(OH)2D3 is more effective. In fact, Zhang et al. (30) reported that combination therapy with ARB and vitamin $\mathrm{D}$ analog markedly ameliorates diabetic nephropathy in streptozotosin treated diabetic mice. However, the mechanisms have not been fully determined in type 2 diabetic nephropathy.

Ohara et al. (31) examined the preventive effects of combination therapy with an ARB and 1,25(OH)2D3 in diabetic nephropathy of KK-Ay mice. KK-Ay mice were divided into four groups as follows: ARB group, 1,25(OH)2D3 group, combination group and control group. The urinary ACR was measured for phenotypic characterization. Renin, pERK1/2 and TGF-b1 protein expressions in the renal tissues were evaluated. The levels of urinary ACR in the combination group were significantly lower than those in the ARB group or control group. Renin protein expressions on renal tissues in the ARB group were significantly increased compared with those found in the control group. In the $1,25(\mathrm{OH}) 2 \mathrm{D} 3$ group or combination group, renin protein expressions were significantly decreased compared with those in the ARB group. The protein expressions of p-ERK1/2 in the combination group were significantly decreased compared with those in the control group or ARB group. The protein expressions of TGF-b1 in the ARB group and/or combination groups, especially the combination group, were significantly decreased compared with those of the control group. It appears that combination therapy with ARB and 1,25(OH)2D3 improves the levels of urinary ACR by suppressing the compensatory renin increase in type 2 diabetic nephropathy. These effects might be related to the suppression of renin-ERK1/2-TGFb1, which may or may not depend on angiotensin II (31).

Clinically, Zeeuw et al. (17) reported that the addition of $2 \mu \mathrm{g} /$ day paricalcitol to renin angiotensin aldosterone system (RAAS) inhibition safely lowers residual albuminuria in patients with diabetic nephropathy, and this could be a novel approach to lowering the residual renal risk in diabetes.

\subsection{Anti-Microinflammatory Effect of Eicosapentaenoic Acid (EPA)}

Previous studies reported that eicosapentaenoic acid (EPA) was effective against all renal diseases including diabetic nephropathy. EPA is one of the n-3 polyunsaturated fatty acids (PUFA) present in fish oil. It was found that EPA has many effects including anti-thrombotic, hypolipidemic, anti-atherogenic, anti-inflammatory and anti-mitogenic actions. Monocyte chemoattractant pro- tein-1 (MCP-1) is a regulating macrophage recruitment protein, which is up-regulated in patients with diabetic nephropathy. In KK-Ay mice injected with EPA ethyl ester (1g/kg/day), Zhang et al. (32) and Hagiwara et al. (30) reported that EPA improved type 2 diabetic nephropathy in KK-Ay mice by decreasing hypertriglyceridemia, glucose tolerance and albuminuria. Glomerular mesangial matrix expansion and segmental sclerosis, as well as interstitial fibrosis were markedly decreased by EPA treatment. Diabetes induced up-regulation of MCP-1 and TGFbeta expressions were inhibited by EPA, together with a reduction of glomerular macrophage infiltration and oxidative stress. It appears that EPA might be an effective therapeutic agent for diabetic nephropathy (33).

Clinically, Lee et al. (34) examined the association between dietary n-3 long-chain polyunsaturated fatty acids (n-3LC-PUFAs), incident albuminuria and changes in urinary albumin excretion rates (UAER) over time in type 1 diabetes. Dietary n-3LC-PUFAs appear to be inversely associated with the degree but not with the incidence of albuminuria in type 1 diabetes.

In conclusion, it appears that KK-Ay mice are a useful spontaneous animal model for the evaluation of pathogenesis and treatment in patients with type 2 diabetic nephropathy. Confirmation that the treatments studied in the KK-Ay mouse are effective in patients with type 2 diabetic nephropathy will be necessary in the future.

\section{Acknowledgments}

I sincerely thank my colleagues of Diabetic Nephropathy Research Group (Leader: Associate Professor Tomohito Gohda and Associate Professor Yoshio Shimizu in the Division of Nephrology, Department of Internal Medicine at Juntendo University Faculty of Medicine, Tokyo, Japan.

\section{Financial Disclosure}

None declared.

\section{Funding/Support}

None declared.

\section{References}

1. Kondo K, Nozawa K, Tomita T, Ezaki K. Inbred strains resulting from Japanese mice. Bull Exp Anim. 1967;6:107-12.

2. Taketomi S, Ikeda H, Ishikawa E, Iwatsuka H. Determination of overall insulin sensitivity in diabetic mice, KK. Horm Metab Res. 1982;14(1):14-8.

3. Matsuo T, Shino A. Induction of diabetic alterations by goldthioglucose-obesity in KK,ICR and C57BL mice. Diabetologia. 1972;8(6):391-7.

4. Ikeda H. KK mouse. Diabetes Res Clin Pract. 1994;24 (Suppl):S313-6.

5. Nishimura M. Breeding of mouse strains for diabetes mellitus. Exp Anim. 1969;18:147-57.

6. Tomino Y. Lessons from the spontaneous mouse models for treatment of type 2 diabetic nephropathy and IgA nephropathy. Juntendo Med J. 2009;55:228-34. 
7. Ito T, Tanimoto M, Yamada K, Kaneko S, Matsumoto M, Obayashi $\mathrm{K}$, et al. Glomerular changes in the KK-Ay/Ta mouse: a possible model for human type 2 diabetic nephropathy. Nephrology (Carlton). 2006;11(1):29-35.

8. Hishiki T, Shirato I, Takahashi Y, Funabiki K, Horikoshi S, Tomino Y. Podocyte injury predicts prognosis in patients with iga nephropathy using a small amount of renal biopsy tissue. Kidney Blood Press Res. 2001;24(2):99-104.

9. Lemley KV, Lafayette RA, Safai M, Derby G, Blouch K, Squarer A, et al. Podocytopenia and disease severity in IgA nephropathy. Kidney Int. 2002;61(4):1475-85.

10. Macedo CS, Lerco MM, Capelletti SM, Silva RJ, Pinheiro Dde O, Spadella CT. Reduction of podocytes number in late diabetic alloxan nephropathy: prevention by glycemic control. Acta Cir Bras. 2007;22(5):337-41.

11. Aoki T, Kaneko S, Tanimoto M, Gohda T, Hagiwara S, Murakoshi $\mathrm{M}$, et al. Identification of quantitative trait loci for diabetic nephropathy in KK-Ay/Ta mice. J Nephrol. 2011:0.

12. Singh R, Barden A, Mori T, Beilin L. Advanced glycation end-products: a review. Diabetologia. 2001;44(2):129-46.

13. Khalifah RG, Baynes JW, Hudson BG. Amadorins: novel post-Amadori inhibitors of advanced glycation reactions. Biochem Biophys Res Commun. 1999;257(2):251-8.

14. Degenhardt TP, Alderson NL, Arrington DD, Beattie RJ, Basgen JM, Steffes MW, et al. Pyridoxamine inhibits early renal disease and dyslipidemia in the streptozotocin-diabetic rat. Kidney Int. 2002;61(3):939-50.

15. Tanimoto M, Gohda T, Kaneko S, Hagiwara S, Murakoshi M, Aoki $\mathrm{T}$, et al. Effect of pyridoxamine (K-163), an inhibitor of advanced glycation end products, on type 2 diabetic nephropathy in KK$\mathrm{A}(\mathrm{y}) /$ Ta mice. Metabolism. 2007;56(2):160-7.

16. Murakoshi M, Tanimoto M, Gohda T, Hagiwara S, Ohara I, Toyoda $\mathrm{H}$, et al. Pleiotropic effect of pyridoxamine on diabetic complications via CD36 expression in KK-Ay/Ta mice. Diabetes Res Clin Pract. 2009;83(2):183-9.

17. de Zeeuw D, Agarwal R, Amdahl M, Audhya P, Coyne D, Garimella $\mathrm{T}$, et al. Selective vitamin $\mathrm{D}$ receptor activation with paricalcitol for reduction of albuminuria in patients with type 2 diabetes (VITAL study): a randomised controlled trial. Lancet. 2010;376(9752):1543-51.

18. Williams ME, Bolton WK, Khalifah RG, Degenhardt TP, Schotzinger RJ, McGill JB. Effects of pyridoxamine in combined phase 2 studies of patients with type 1 and type 2 diabetes and overt nephropathy. Am J Nephrol. 2007;27(6):605-14

19. Yamazaki T, Tanimoto M, Gohda T, Ohara I, Hagiwara S, Murakoshi M, et al. Combination effects of enalapril and losartan on lipid peroxidation in the kidneys of KK-Ay/Ta mice. Nephron Exp Nephrol. 2009;113(2):e66-76.

20. Jennings DL, Kalus JS, Coleman CI, Manierski C, Yee J. Combination therapy with an ACE inhibitor and an angiotensin receptor blocker for diabetic nephropathy: a meta-analysis. Diabet Med. 2007;24(5):486-93.

21. Huang Y, Wongamorntham S, Kasting J, McQuillan D, Owens $\mathrm{RT}$, Yu L, et al. Renin increases mesangial cell transforming growth factor-beta1 and matrix proteins through receptormediated, angiotensin II-independent mechanisms. Kidney Int. 2006;69(1):105-13.

22. Huang Y, Noble NA, Zhang J, Xu C, Border WA. Renin-stimulated TGF-beta1 expression is regulated by a mitogen-activated protein kinase in mesangial cells. Kidney Int. 2007;72(1):45-52

23. Kaneshiro Y, Ichihara A, Sakoda M, Takemitsu T, Nabi AH, Uddin $\mathrm{MN}$, et al. Slowly progressive, angiotensin II-independent glomerulosclerosis in human (pro)renin receptor-transgenic rats. J Am Soc Nephrol. 2007;18(6):1789-95.

24. Kuhlmann A, Haas CS, Gross ML, Reulbach U, Holzinger M, Schwarz U, et al. 1,25-Dihydroxyvitamin D3 decreases podocyte loss and podocyte hypertrophy in the subtotally nephrectomized rat. Am J Physiol Renal Physiol. 2004;286(3):F526-33.

25. Schwarz U, Amann K, Orth SR, Simonaviciene A, Wessels S, Ritz E. Effect of 1,25 (OH)2 vitamin D3 on glomerulosclerosis in subtotally nephrectomized rats. Kidney Int. 1998;53(6):1696-705.

26. Makibayashi K, Tatematsu M, Hirata M, Fukushima N, Kusano K, Ohashi S, et al. A vitamin D analog ameliorates glomerular injury on rat glomerulonephritis. Am J Pathol. 2001;158(5):1733-41.

27. Li YC, Qiao G, Uskokovic M, Xiang W, Zheng W, Kong J. Vitamin $\mathrm{D}$ : a negative endocrine regulator of the renin-angiotensin system and blood pressure. J Steroid Biochem Mol Biol. 2004;89-90(15):387-92.

28. Yuan W, Pan W, Kong J, Zheng W, Szeto FL, Wong KE, et al. 1,25-dihydroxyvitamin D3 suppresses renin gene transcription by blocking the activity of the cyclic AMP response element in the renin gene promoter. J Biol Chem. 2007;282(41):29821-30.

29. Panichi V, Migliori M, Taccola D, Filippi C, De Nisco L, Giovannini $\mathrm{L}$, et al. Effects of 1,25(OH)2D3 in experimental mesangial proliferative nephritis in rats. Kidney Int. 2001;60(1):87-95.

30. Zhang Z, Zhang Y, Ning G, Deb DK, Kong J, Li YC. Combination therapy with AT1 blocker and vitamin D analog markedly ameliorates diabetic nephropathy: blockade of compensatory renin increase. Proc Natl Acad Sci U S A. 2008;105(41):15896-901.

31. Ohara I, Tanimoto M, Gohda T, Yamazaki T, Hagiwara S, Murakoshi $\mathrm{M}$, et al. Effect of combination therapy with angiotensin receptor blocker and 1,25-dihydroxyvitamin $\mathrm{D}(3)$ in type 2 diabetic nephropathy in KK-A(y)/Ta mice. Nephron Exp Nephrol. 2011;117(4):e124-32.

32. Zhang M, Hagiwara S, Matsumoto M, Gu L, Tanimoto M, Nakamura S, et al. Effects of eicosapentaenoic acid on the early stage of type 2 diabetic nephropathy in KKA(y)/Ta mice: involvement of anti-inflammation and antioxidative stress. Metabolism. 2006;55(12):1590-8.

33. Hagiwara S, Makita Y, Gu L, Tanimoto M, Zhang M, Nakamura S, et al. Eicosapentaenoic acid ameliorates diabetic nephropathy of type 2 diabetic KKAy/Ta mice: involvement of MCP-1 suppression and decreased ERK1/2 and p38 phosphorylation. Nephrol Dial Transplant. 2006;21(3):605-15.

34. Lee CC, Sharp SJ, Wexler DJ, Adler AI. Dietary intake of eicosapentaenoic and docosahexaenoic acid and diabetic nephropathy: cohort analysis of the diabetes control and complications trial. Diabetes Care. 2010;33(7):1454-6. 